\title{
Panorama de las vías de comunicación en Navarra durante la Alta Edad Media (siglos VIII-XII)
}

\author{
F. Javier Villalba Ruiz de Toledo*
}

\begin{abstract}
El papel de Navarra en la primera mitad del siglo XI es de una relevancia incostestable para el posterior desarrollo y evolución de los reinos cristianos de la Península Ibérica. Es comúnmente aceptado, asímismo, que la conexión del reino de Navarra de la mano de Sancho 111 con el occidente de Europa supone una de las claves para entender debidamente este proceso. Tal asociación necesitó, lógicamente, de una infraestructura vial que lo permitiera, y sólo a través de su sistemático conocimiento estaremos en condiciones de entender e interpretar correctamente la andadura peninsular hacia la plenitud medieval.

El planteamiento, por tanto, justifica sobradamente el espectro cronológico que hemos escogido para nuestro trabajo. Sólo con un recorrido previo por la realidad vial de Navarra anterior a la transformación señalada, es posible adentrarnos con éxito en la averiguación de las claves de ésta. La nueva toma de conciencia y valoración expresa de los monarcas por el buen funcionamiento y ampliación de las vías de comunicación, ya en los albores del siglo XIII ${ }^{1}$ con la modificación que ello determina en las mismas, nos induce a detenernos en dicho punto, pues a partir de entonces la propia orientación de su estudio exige un planteamiento acorde con las nuevas directrices oficiales.
\end{abstract}

Por consiguiente, y al objeto de valorar con precisión la transformación estructural que impulsa Sancho el Mayor, debemos contemplar la realidad

\footnotetext{
* Universidad Autónoma de Madrid.

El ejemplo más notable y conocido es la inclusión de algunos párrafos sobre el particular en las «Partidas» de Alfonso X. En concreto vid. Segunda Partida, Título XI: "Qual deue el Rey ser a su tierra", Ley I: «.... deuen mandar labrar las puentes, e las calçadas, e allanar los passos malos, por que los omes puedan andar, e lleuar sus bestias, e sus cosas, desembargadamente, de vn lugar a otro, de manera que las non pierdan en los passajes de los rios, ni en los otros lugares peligrosos, por do fueren...".
} 
geográfica del reino de Navarra a partir de la dominación musulmana de la Peninsula -a fin de reconocer la materia prima de la que luego surge el producto terminado - y continuar nuestra labor hasta el momento de la reconducción oficial atribuible ya al conjunto de los reinos cristianos de la Peninsula.

Aunque compuestos en el siglo XVI, los clásicos repertorios de caminos de Villuga y Meneses ${ }^{2}$ son absolutamente imprescindibles para nuestro acercamiento a la realidad vial en la Edad Media. De alguna manera representan el punto de llegada de la politica medieval en torno a las vías de comunicación. Por ello, y centrándonos en la mejor estructurada de Alonso de Meneses, debemos en primer lugar reproducir los caminos que el mismo señala para Navarra. El primero de ellos, de Burgos para Barcelona describe el tramo Burgos-Logroño del Camino de Santiago, y desde la capital riojana atraviesa el sur de Navarra desde las inmediaciones de Calahorra hasta Tudela ${ }^{3}$. Por el norte, Meneses aborda el espacio geográfico de Navarra desde Roncesvalles en el itinerario que denomina Santiago para San Juan de Pie de Puerto. En el mismo, siguiéndolo en el sentido inverso al descrito, se une el paso pirenaico con Pamplona ${ }^{4}$.

Desde allí existe la doble variante entre seguir a Logroño embocando la ruta principal de los peregrinos a Santiago, o bien descender hacia el Sur, hacia Tudela, atravesando en el paso la localidad de Olite por medio del tramo que Meneses denomina Pamplona para Tudela de Navarra ${ }^{5}$.

Finalmente, el sector oriental navarro se halla atravesado, según este repertorio, por el camino que desde Olite parte hacia Monzón ${ }^{6}$.

2 VILluga, P.J. Repertorio de todos los caminos de España compuesto por Pedro Juan Villuga (1546). Ed. facsimil, Madrid, 1950; MENESES, A. de. Repertorio de caminos, ordenado por Alonso de Meneses, Correo (1576). Ed. facsimil, Madrid, 1946.

3 Desde Calahorra, el itinerario continúa por La aldea (Aldeanueva de Ebro), Alfaro, y ya en Navarra, Tudela y Cortes, recorriendo entre la primera y la última localidad un total de 10 leguas, es decir, unos 65 kilómetros, Meneses, fols. VI r-v.

4 La etapa del camino, siempre en el sentido contrario al propuesto por Meneses, sería Roncesvalles, Burguete, Subiri (Zubiri), Rasnay, Villalva (Villava) y Pamplona, con una distancia total de 13 leguas. Ibid. fol. XXXVIII.

5 Con las siguientes etapas: Trevas (Tievas), Varasnay (Barasoain), Tafalla, Olite, Paroso (Caparroso), Santa María del Pucijo, Arguedas y Tudela; 16 leguas en total y una venta localizada entre Tievas y Barasoain. Ibid. fol. XXXI.

- Designado como camino de Pamplona para Monzón y del que nos interesan las localidades de Teyre (Beire), Morielo (Murillo) y Carcastillo. Sin embargo hay que consignar una pequeña variación del tramo que previamente une Pamplona y Olite, en el que su arranque se diferencia del itinerario anterior. Comienza este segundo por Asnoa para continuar por Tievas, Arte de Reta (Artariain?), Varasunay (Barasoain) y el resto del recorrido ya es idéntico. Ibid. fols. XXXI r-V. En 
De todas ellas, sólo tenemos constancia de superposición con vías romanas en cuanto a la meridional que desde Calahorra parte en dirección a Zaragoza atravesando Tudela ${ }^{7}$, lo que indica un desarrollo auténticamente medieval de las vías de comunicación navarras. Así parece constatarlo, al menos, la lectura del Codex Calixtinus ${ }^{8}$, según el cual la vía «tolosana» del Camino de Santiago ${ }^{9}$ suponía un nuevo incremento de la red viaria romana que atravesaba de Este a Oeste el reino de Navarra para unirse a la célebre final confluencia de Puente la Reina. El tramo que uniría en el siglo XVI el paso de Roncesvalles con Pamplona, se halla entonces en perfecto estado de funcionamiento, y aunque podemos presumir su mayor antigüedad, lo cierto es que no podemos ver en él la mano de la ingeniería romana.

Pero si bien es verdad que estos son los datos fundamentales con los que podemos empezar a reconstruir la red viaria de Navarra en la etapa propuesta, está claro que con ello no nos hemos sino acercado vagamente a la realidad que nos interesa. Una extensa labor de archivo y la confrontación de los datos que alli nos aparezcan, será la única forma de reconstruir realmente el entramado de caminos por el que hombres, ideas y mercancías cubririan la realidad espacial cotidiana del reino. Por el momento, sin embargo, nos conformaremos con ofrecer una valoración de lo hasta hoy aportado por los numerosos investigadores que directa o tangencialmente han abordado el tema de las vías de comunicación medievales en Navarra, así como a señalar las fuentes y colecciones documentales más elementales para la reconstrucción que proponemos. Algunos trabajos sobre la situación de los caminos navarros del siglo XVIII en adelante ${ }^{10}$, representan un punto de apoyo fundamental en nuestro trabajo.

Sería inútil abordar cualquier investigación sobre el espacio geográfico de la Navarra medieval sin acudir en primera instancia a las magníficas obras de síntesis que sobre la Historia del Reino publicara el profesor

definitiva, la única diferencia real entre ambos itinerarios es la inclusión de dos nuevas etapas en este segundo.

7 En concreto se corresponde con la Vía A 32 del Itinerario de Antonino. Vid. ARIAS, G. Repertorio de caminos de la Hispania romana, Madrid, 1987; Roldán Hervas, J.M. Itineraria Hispana. Fuentes antiguas para el estudio de las vías romanas en la Península lbérica, ValladolidGranada, 1975 y obras generales acerca del entramado viario peninsular en época romana.

\& Vid. por ejemplo, Guía del Peregrino Medieval ("Codex Calixtinus»), Trad. M. Bravo Lozano, Sahagún, 1989; VIELLIARD, J. Le Guide du Pélerin de Saint-Jacques de Compostelle, 1978.

9 Desaparecida en los itinerarios de Villuga y Meneses.

10 Vid. por ejemplo "La construcción de nuevos caminos carretiles en Navarra, 1750-1784", Príncipe de Viana, agosto-diciembre, 1985. 
Lacarra, tanto su obra de conjunto ${ }^{11}$ como algunos estudios parciales, generalmente orientados hacia el conocimiento de una etapa posterior ${ }^{12}$, pero enormemente ricos en datos que han de ser de nuestro más alto interés. Un acercamiento al soporte documental con que podemos contar en nuestro trabajo, nos fue servido ya hace varias décadas por el propio Lacarra en sus Documentos navarro-aragoneses ${ }^{13}$ y en los Documentos para estudio de la reconquista y repoblación del valle del Ebro ${ }^{14}$. Otras colecciones documentales absolutamente imprescindibles serían las de Santa María de Roncesvalles ${ }^{15}$, Leire ${ }^{16}$ e Irache ${ }^{17}$, y los catálogos de Comptos ${ }^{18}$ y Cartularios reales ${ }^{19}$ del Archivo General de Navarra, así como los referentes a archivos municipales.

El estudioso de Navarra, no obstante, no puede conformarse con la consulta de obras centradas en su geografía. Así, y aunque necesariamente de forma breve, comentaremos algunos estudios de espectro espacial peninsular a los que hay que acudir reiteradamente en este tipo de investigaciones. Destacaremos entre ellos las rutas medievales de García Piñol ${ }^{20}$, los caminos en la Historia de España de G. Menéndez Pidal ${ }^{21}$, y desde luego obras como el Panorama de la historia urbana de J.M. Lacarra ${ }^{22} \mathrm{O}$ el Itinerario de la conquista musulmana de C. Sánchez-

11 En la que analiza las rutas comerciales desde el siglo XII al XIII, especialmente en conexión con la aparición o desarrollo de ciertos núcleos urbanos. Por otra parte, y entre los siglos XIII y XIV, se detiene en la conocida lucha de Navarra por obtener una salida al mar tras el avance territorial del reino de Castilla al anexionarse Guipúzcoa. LACARRA, J.M. Historia del reino de Navarra en la Edad Media, Pamplona, 1975, pp. 255-257; 333-334.

12 Id. "Estructura económica y social del reino de Navarra en el siglo XIV", Cuadernos de Historia, Anexos de la revista Hispania, VIII, Madrid, 1977, pp. 227-236; id. "Las cortes de Aragón y de Navarra en el siglo xiv", Anuario de Estudios Medievales, I, Simposio de Historia Medieval, VII, Barcelona, 1970, pp. 645-652. No podemos olvidar, desde luego, el trabajo del profesor Lacarra sobre Rutas de peregrinación. Los pasos del Pirineo y el camino de Santa Cristina a Puente la Reina, Pirineos, año 1, N², 1945.

13 Id. "Documentos navarro-aragoneses", Anuario de Historia del Derecho Español, XVIII, Madrid, 1947, pp. 341-347.

$14 \quad 2^{\mathrm{a}}$ edición, Zaragoza, 1982-85.

15 Ostolaza, Ma I. Colección Diplomática de Santa María de Roncesvalles (1127-1300), Institución Príncipe de Viana.

16 Martín Duque, A.J. Documentación medieval de Leire (siglos IX-XII), Pamplona, 1983.

17 Lacarra, J. M. Colección Diplomática de Irache. Vol. I (958-1222), Zaragoza, 1965.

18 Baleztena, J., Castro, J. R. e looate, F. Catálogo del Archivo General de Navarra. Sección de Comptos, Pamplona, 1952-1985.

19 IDOATE, F. Catálogo de los cartularios reales del A.G.N., Anuario de Historia del Derecho Español, X, 1933, pp. 203-272.

20 Garcia PINOL, R. Rutas medievales, Madrid, 1974.

21 Menéndez Pidal, G. Los caminos en la Historia de España, Madrid, 1951.

22 Lacarra, J. M. «Panorama de la historia urbana en la Peninsula Ibérica desde el siglo $\mathrm{V}$ hasta el X", Settimane de Studio de Spoleto, 1959, pp. 319-357. 
Albornoz ${ }^{23}$. Y por supuesto, no podemos arrinconar el importante coloquio que dedicado a las vías de comunicación medievales en la Península Ibérica, se desarrolló en la Universidad de Pau en $1980^{24}$. Recientemente se han publicado también las actas del Coloquio sobre la circulación de códices entre la Península Ibérica y la Europa medieval ${ }^{25}$, que junto a los datos que nos proporcionan sobre la distribución geoespacial de la red viaria española, nos describe las distintas maneras de concebir un viaje y como ello influye en otros aspectos del desarrollo histórico de las comunidades que se analizan. Por último, y aunque tangencialmente, se abordan las vias de comunicación medievales en algunos de los trabajos que integran el VI Congreso Español de Historia del Arte, cuyo subtítulo - los caminos y el arte- determinan claramente su línea de investigación principal.

Asimismo, los estudios criticos de libros o documentos de viajes son de vital importancia para nosotros. Pensemos, por poner sólo un ejemplo, en la edición llevada a cabo por $\mathrm{M}^{\mathrm{a}} \mathrm{D}$. Sánchez Villar de las cuentas del viaje de Estella a Sevilla de $1352^{26}$.

Pero descendamcs ya a lo concreto y analicemos el estado de la investigación sobre vias de comunicación en Navarra durante la Alta Edad Media. Desde luego no existe, como para otras áreas peninsulares, ninguna obra de conjunto que aborde frontalmente el tema. Al ser Navarra paso obligado del Camino de Santiago, es en los numerosos trabajos sobre el mismo ${ }^{27}$ donde se enfoca el tema con índices más o menos elevados de análisis sobre el conjunto del territorio navarro. Tal vez podamos destacar como aportación singular en este sentido el trabajo de Urrutibehety ${ }^{28}$ en el que se describe la unión de la ruta jacobea con los caminos de Navarra a partir del estudio de las "Guías del Peregrino" pu-

\footnotetext{
23 SÁnchez Albornoz, C. “ltinerario de la conquista de España por los musulmanes», Origenes de la Nación Española, I, Oviedo, 1972, pp. 413-458.

24 Les communications dans la Peninsule Iberique au Moyen Age, Actes Colloque Pau 28-29 mars, 1980.

${ }_{25}$ Coloquio sobre circulación de códices y escritos entre Europa y la Peninsula lbérica en los siglos VIII-XIII, Santiago, 1988.

26 SÁnChez VILlar, Mª D. Desde Estella a Sevilla. Cuentas de un viaje (1352), Valencia, 1962.

27 Piénsese, por ejemplo en las obras de E. Golcoechea Arrondo, Cartografia del Camino de Santiago, Estella, 1972; Y. BotTINEAU, Les chemins de Saint-Jacques, París, 1983; L. HuIDobro $r$ SERnA, Las peregrinaciones jacobeas, Madrid, 1951; E. Valiña SAMPEDRO, El camino de Santiago. Estudio histórico-jurídico, Madrid, 1971; o L. VázQuez de PARga, J. M. Lacarra, y J. Uría Riu, Las peregrinaciones a Santiago de Compostela, Madrid, 1949.

28 URRutiBehetY, C. "Union des chemins de Saint-Jacques en Basse-Navarre et en Navarre", Príncipe de Viana. Primer Congreso General de Historia de Navarra, XLIX, 1988, pp. 207-217.
} 
blicadas hasta 1982. Uno de los itinerarios mejor estudiados es el llamado Camino de Euñate o Camino de Eneriz a Puente.

Contamos, no obstante, con un buen número de trabajos que presentados en el Primer Congreso General de Historia de Navarra, nos proporcionan gran cantidad de datos útiles para la reconstrucción que intentamos. Así por ejemplo, Anchon Insausti centra sus colaboraciones en los problemas fronterizos entre Navarra y Guipúzcoa ${ }^{29}$, y aunque inscritos sus trabajos en el siglo $\mathrm{xV}$, nos describe el espacio geográfico cercano a Tolosa en el momento en que esta ciudad busca una salida al mar y un mayor control sobre su entorno rural inmediato. Como quiera que Tolosa era punto de unión entre el Camino de Santiago y San Sebastían y parte integrante del tramo de la ruta de la lana que unía Pamplona con San Sebastián, su relevancia comercial provocó el nacimiento de dos facciones rivales - hidalgosnavarros y banderizos - que a la postre derivó en la apertura de vías alternativas al paso por la ciudad, lo que sin duda enriqueció el panorama viario de la zona. A partir de ahí es posible deducir el armazón según el cual se escogeria uno u otro camino como respuesta a dicha alternativa.

En la línea de los estudios centrados en los peajes navarros, ceñidos, desde luego, a los siglos bajomedievales, destacaremos por su aportación al estudio de los caminos el trabajo de J. Carrasco ${ }^{30}$ acerca del peaje

29 Achón insausti, J. A. y De Orella Unzúe, J. L. «Los intereses de la villa de Tolosa en la frontera navarro-guipuzcoana", Principe de Viana. Primer Congreso General de Historia de Navarra, XLIX, 1988, pp. 267-275; Ibid. "Los intereses banderizos en la definitiva configuración de la frontera entre Guipúzcoa y el Reino de Navarra», pp. 257-265; Ibid. y SAIz ELIzONDO, P.J. “LoS amojonamientos de los términos jurisdiccionales de Rentería, Oyarzun y Fuenterrabia en la segunda mitad del siglo xv: La conclusión de un proceso de delimitación espacial en la frontera entre Guipúzcoa", pp. 277-286; Vid. asimismo para el panorama de la politica fronteriza de Navarra, Munita LoINAz, J. A. «Intereses político-estratégicos de Carlos II en Alava y Guipúzcoa: el tratado de Liborne (1366)", La formación de Alava, Vol. II, pp. 763-775, CIERBIDE, R. "Conflictos fronterizos entre Navarra, Guipúzcoa y Alava en el siglo XIV", Vitoria en la Edad Media, Vitoria, 1982, y respecto a la frontera con La Rioja, P. AZCÁRATE AguILAR-AMAT nos habla de las "Hostilidades en la frontera navarro-riojana durante el siglo xIV: el choque de los años 1344-45", I/ Coloquio de Historia de La Rioja, Logroño, 1985, pp. 333-343, centrándose en los entrentamientos producidos entre la villa riojana de Alfaro y las navarras de Corella, Cintruénigo y Tudela por el aprovechamiento de las aguas del rio Alhama. Al tratar el asunto, la autora nos describe el camino que une Tudela con Santo Domingo (escenario de una procesión inscrita en el conflicto), así como el viaje realizado por un baile y un merino riojanos para entrevistarse con Alfonso XI de Castilla como maximo árbitro de la situación. El camino hacia Madrid podemos suponerlo como poco dotado de medidas de seguridad, a tenor de la comitiva que necesitan estos personajes en su desplazamiento "por el recelo de las malas genetes que andauan por el camino" (A.G.N. Reg. 54 , fols. $48 \mathrm{r} .-62 \mathrm{~V}$.).

30 Carrasco Pérez, J. «Documentos para el estudio de las aduanas bajomedievales: el peaje de Pamplona de 1358", Cuadernos de Estudios Medievales, VIII-IX, Granada, 1980-81, pp. 109-155. 
de Pamplona de 1358 y el reciente artículo de Grocin Gabas ${ }^{31}$ a partir del análisis de los registros contables correspondientes al reinado de Carlos II. Se nos ofrece aqui una relación de los productos recogidos en estos peajes, y sobre todo, una estadística con las épocas en que es más frecuente la aparición de tales productos en el peaje de Pamplona. De esta forma es posible, estudiando su procedencia y destino, determinar los itinerarios comerciales subsiguientes para cada uno de estos objetos de intercambio. Algo parecido ocurre con el trabajo de Herrero y Orella ${ }^{32}$, donde se establece la relación entre la situación política y social de Navarra y Guipúzcoa, así como sus relaciones comerciales en los siglos XIV y XV. Los autores, que parten de la premisa según cual las relaciones comerciales entre ambas regiones está en conexión directa con sus relaciones políticas, llegan a la conclusión de que ambos, a su vez, influyen decisivamente en la utilización de las vías de comunicación, que con una alta actividad mercantil, permiten a Navarra una salida al mar para sus productos. Se presentan, para esta etapa cronológica, dos vías fundamentales: la que llegaba a San Sebastián por Tolosa - que hacía inevitable el paso por una región fronteriza y conflictiva en la que eran abundantes los peajes a satisfacer-, y la que alcanzaba Fuenterrabía por el puerto de Belate y el valle de Bidasoa, es decir, agotando al máximo el territorio navarro en su recorrido ${ }^{33}$. La seguridad que ofreciera cada una de ellas se revela como el argumento fundamental que esgrimen los comerciantes en su opción particular, lo que coincide con esa inquietud generalizada de la monarquía que, por estas fechas, no lo olvidemos, apostaba decididamente por la seguridad de los caminos, y desde luego Navarra no podía ser una excepción. También aquí seguridad vial era sinónimo de auge mercantil, y por lo tanto su salvaguarda era algo conceptuado como prioritario ${ }^{34}$. Otros estudios relativos a peajes a destacar por su aportación al

${ }^{31}$ Grocin GaBAS, M${ }^{a} \mathrm{C}$. «Análisis comparado de las principales mercancías de los peajes de Pamplona en la $2^{\mathrm{a}}$ mitad del siglo XIV", Príncipe de Viana. Primer Congreso General de Historia de Navarra, XLIX, 1988, pp. 457-466.

32 Herrefo Liceaga, V. y de Orella UnzúE, J. L. «Las relaciones comerciales entre Navarra y Guipúzcoa desde mediados del siglo XIV hasta mediados del siglo XV", Príncipe de Viana. Primer Congreso General de Historia de Navarra, XLIX, 1988, pp. 491-500.

${ }_{33}$ Ibid. pp. 495-496. Al margen de ofrecemos esta valoración acerca de los itinerarios, quedan perfectamente documentados los mismos en virtud de una relación pormenorizada de las noticias que contienen de forma especial ciertos privilegios reales, tanto navarros como castellanos.

${ }^{34}$ Sin duda ese va a ser el motivo de la aparición de las Hermandades de frontera e incluso de la apertura o rehabilitación de varios caminos de entrada al territorio de Guipúzcoa con objeto de conjurar un posible colapso originado por la actividad bélica desarrollada en la zona. Para el estudio de las hermandades en Navarra podemos acudir a los trabajos de ÁlVAREZ DE MORALES, A. "La Hermandad de Vitoria, Alava y Guipúzcoa, Val de Lana y otros en defensa del reino de Navarra 
conocimiento de las vías de comunicación serían los de A. Martín Duque ${ }^{35}$ analizando los casos de Vera y Lesaca, de C. Grocin ${ }^{36}$ y J. Llanso ${ }^{37}$ acerca del Peaje de Pamplona, y las distintas aportaciones de J. Zabalo sobre los peajes en Tudela, Sanguesa, Carcastillo y la propia Pamplona ${ }^{38}$.

Junto a la generalizada protección de los caminos, también Navarra participaba ahora del interés por su conservación material. En este sentido, algunos acuerdos conjuntos pactados con Castilla-Guipúzcoa parecen afirmarlo ${ }^{39}$. Resulta interesante la comparación de las características aduaneras de Navarra durante la Baja Edad Media con respecto a las estimables para la época altomedieval. J. M. Lacarra ${ }^{40}$ nos ofrece una vision ajustada de las mismas en el siglo XI, donde, evidentemente, se detecta un desarrollo porcentualmente muy inferior del entramado viario que sirve de marco a las transacciones mercantiles. Muy útil para esta valoración arancelaria resulta el trabajo de J. Llanso ${ }^{41}$ que intenta reconstruir el arancel del peaje de Tudela apoyándose en los datos comparativos que le ofrecen los ejemplos de Alagón y Gallur. Tampoco hemos de olvidar en este apartado la colaboración de E. Ochoa de Alza ${ }^{42}$, quien analiza los productos más comunes en los aranceles de peajes navarros de Sangüesa -comercio con Aragón-, los Arcos y Sesma - que controlan las importaciones desde Castilla-, Maya-Ainhoa - control del comercio con Francia-y Pamplona, en donde confluye el comercio francés.

(1368-1369)", Vitoria en la Edad Media. Actas del Congreso de 1981, Vitoria, 1982, pp. 341-347; INSAUSTI, S. «Tolosa en la formación de la Hermandad guipuzcoana», Tolosa, 1969; ORELLA, J. L. "La Hermandad de frontera entre Navarra y Guipúzcoa en los siglos XIV y XV», Príncipe de Viana, 46. 175; Id. "Los origenes de la Hermandad de Guipúzcoa (las relaciones Guipúzcoa-Navarra en los siglos XIII y XIV)". Cuadernos de la Sección Historia-Geografia, 3, San Sebastián, 1984, pp. 25-100.

35 Martin Duque, A. J. y otros, «Peajes navarros. Vera y Lesaca (1358-1359)», Homenaje a J. E. Uranga, Pamplona, 1971, pp. 69-86.

36 Grocin Gabas, C. "Peaje de Pamplona de 1354", Memoria de Licenciatura, marzo, 1986.

37 Llanso Sanjuán, J. "Peaje de Pamplona de 1362", Memoria de Licenciatura, junio, 1986.

sя Zabalo Zabalegul, J. «Peaje de Pamplona (1355)», Príncipe de Viana, 46, 1985, pp. 675722; "Peajes navarros. Carcastillo (1357). Tudela (1380), Sangüesa (1380)", Pamplona, 1972; "Peajes navarros. Pamplona (1351), Tudela (1365), Sangüesa (1362), Carcastillo (1362)", Pamplona, 1973.

39 Sabemos, por ejemplo, del intento por adecentar la via de Fuenterrabia en tiempos de Carlos II, y de la participación navarra en el mismo empeño referido a una serie de caminos que les comunicaban con los puertos guipuzcoanos hacia el año 1414. Incluso contamos con una orden personal de Carlos III fechada el 12 de mayo de 1406 (AGN, Catálogo XXVI, 963) dictada para la obtención de este propósito.

40 LACARRA, J. M. "Un arancel de aduanas del siglo $x \mid »$, Zaragoza, 1950.

41 Llanso Sanjuán, J. «El arancel del peaje de Tudela en la Edad Media. intento de reconstrucción". Principe de Viana. Primer Congreso General de Historia de Navarra, XLIX, 1988, pp. 519-523.

42 Ochoa de Alza Eguiraun, E. «Las mercancias de los aranceles de peaje navarros", Príncipe de Viana. Primer Congreso General de Historia de Navarra, XLIX, 1988, pp. 573-574. 
Otro círculo de conocimiento que nos proporciona abundante información para la reconstrucción vial de la Navarra altomedieval, sería el que integran los estudios poblacionales, con especial atención a aquellos que abordan las relaciones recíprocas de comunidades rurales y de señoríos monásticos o laicos.

Recientemente, E. García Fernández ${ }^{43}$ ha publicado un denso estudio sobre los fundamentos económicos del monasterio de Santa María de Irache en el que se ofrece un panorama completo de los núcleos de producción y abastecimiento con que se nutre el señorío. La conexión entre los distintos lugares geográficos en que se asienta el modelo productivo representa, sin duda ninguna, un buen punto de apoyo para verificar el cauce por el que la misma se producía. En esa misma línea, M. Vaquero ${ }^{44}$, apoyándose en la documentación particular del monasterio de Irache, analiza el paisaje agrario y los cambios que en este se producen durante los siglos XI, XII y XIII, destacando el desarrollo de un proceso según el cual a partir de un policultivo autárquico que únicamente admite la presencia de una red viaria elemental, se evoluciona desde el siglo Xll hacia una concentración del paisaje agrario en torno a las villas y ciudades de más importancia y, desde luego, en torno a las principales vías de comunicación. Junto al Camino de Santiago, las vías de Arvingorría, Agoncello y de Lizagorría, serán las más afectadas por tal concentración. Se trata de un fenómeno recíproco, en tanto en cuanto ello produce a su vez el desarrollo y mejoramiento de las propias vías de comunicación. En cuanto a las razones que mueven a ese acercamiento a las grandes vias comerciales, no puede ser más elemental: se abarataba considerablemente el transporte de los productos agricolas. Por último destacaremos el trabajo de E. Cantera ${ }^{45}$ en que, desde la documentación monástica, se hace un balance cartográfico comparativo entre los lugares de poblamiento riojanos del siglo XIII y los del siglo XVI.

También desde el punto de vista arqueológico se ha avanzado en el estudio de la sociedad rural navarra en la Edad Media. Un buen ejemplo lo hallamos en el trabajo de $\mathrm{C}$. Jusue Simonena ${ }^{46}$, en el que a través de los

\footnotetext{
43 «Santa Maria de Irache: expansión y crisis de un señorío monástico navarro en la Edad Media (958-1537)», Bilbao, 1989.

${ }_{44}$ VAQUERO PIÑEIRO, M. «El paisaje agrario del señorío monástico de Sta. Mª de Irache (9581222). Contribución al estudio del campo navarro de la Alta Edad Media», Príncipe de Viana. Primer Congreso General de Historia de Navarra, XLIX, 1988, pp. 217-223.

45 Cantera montenegro, E. "La red de poblamiento en la Rioja media y baia y en Cameros a mediados del siglo XIII", /I Coloquio de Historia de la Rioja, Logroño, 1985, pp. 315-332.

46 Jusue Simonena, C. «Poblamiento rural en Navarra en la Edad Media. Bases arqueológicas", Pamplona, 1988.
} 
yacimientos analizados, se aportan datos de enorme interés para la comprensión y el entendimiento de la estructura agraria y concentración poblacional de la zona. La misma autora, en su aportación al Primer Congreso de Historia de Navarra ${ }^{47}$ describe el proceso de despoblación -aplicable al conjunto peninsular- que sufren algunas regiones de Navarra a partir del siglo XIV, atribuyendo tal circunstancia a la pérdida de atractivo económico de las mismas en razón directa a la aparición o consolidación de focos más relevantes en este sentido.

B. Leroy ${ }^{49}$, por su parte, aborda el problema desde la doble óptica rural y urbana, analizando el proceso de despoblación que sufren ambos espectros y el subsiguiente relanzamiento de algunos integrantes de ellos. El papel que desempeña la oficialidad en semejante proceso queda, a juicio de este autor, perfectamente probado ${ }^{49}$. Aunque a un nivel muy general, resulta imprescindible la consulta del Gran Atlas de Navarra, que nos ofrece una reconstrucción gráfica de los despoblados navarros por etapas. La que a nosotros nos interesa sería precisamente el reflejo de los despoblados anteriores a $1300{ }^{50}$.

Otra de las aportaciones interesantes sobre el particular nos la ofrece $\mathrm{M}^{\mathrm{a}} \mathrm{A}$. Irurita ${ }^{51}$, quien saca a la luz los acuerdos bilaterales pactados entre los burgos de la Pamplona medieval durante los siglos XIII y XIV como aspecto positivo que contrasta con la tradicional visión que se ha dado centrando su atención en las desavenencias surgidas entre ellos. Una tendencia colectiva hacia la unidad - conclusión central del trabajocondicionaría significativamente las propias relaciones comerciales de tales burgos.

Es bien sabido que el estudio de los fueros representa una valiosísima fuente de información para cualquier reconstrucción de índole socioeconómica en la Edad Media. En el espacio geográfico de Navarra son

47 Ibid. "Arqueologia y desolados medievales". Príncipe de Viana. Primer Congreso General de Historia de Navarra, XLIX, 1988, pp. 99-104.

48 LEROY, B. «Ruina y reconstrucción. Los campos y las ciudades de Navarra en la segunda mitad del siglo Xiv», Hispania, CLVII, Madrid, 1984, pp. 237-261.

49 Vid. Ibid. "La cour des rois de Navarre dans la deuxieme moitié du XIV siècle et au début du XV siècle, une recontre de techniciens". Anuario de Estudios Medievales, estudios dedicados a la memoria de Don Claudio Sánchez Albornoz, II, Barcelona, 1986, pp. 307-318; id. "Un officier des finances du roi de Navarre au milieu du XIV siècle: Lucas Lefèvre". Anuario de Estudios Medievales, Estudios dedicados a la memoria del profesor doctor Emilio Sáez, XVII, Barcelona, 1987, pp. 229-236.

so "Gran Atlas de Navarra", Pamplona, 1986, p. 134.

51 IRURita Lusarieta, Ma A. "Relaciones entre los burgos de la Pamplona medieval», Príncipe de Viana. Primer Congreso General de Historia de Navarra, XLIX, 1988, pp. 505-509. 
numerosos los trabajos acometidos acerca de ellos. Sin entrar en los grandes estudios sobre el Fuero General de Navarra ${ }^{52}$, debemos señalar las aportaciones de algunos estudiosos que han conectado los contenidos de los textos forales con una serie de temáticas estrechamente relacionadas con nuestro objeto de análisis. López Beltrán ${ }^{53}$, por ejemplo, entresacó las ordenanzas y peculiaridades de un factor productivo como el molino, que se contienen en el corpus foral del Valle del Ebro. Del mismo modo, $\mathrm{M}^{\mathrm{a}} \mathrm{A}$. Zamanillo ${ }^{54}$, repasando conjuntamente los textos forales de Navarra y algunos cartularios como los de Leire, Irache o Roncesvalles, lleva a cabo un estudio sobre la circulación monetaria en Navarra durante los siglos $\mathrm{x}$ al XIII, confirmando con el mismo el hecho de que tanto la consolidación del Camino de Santiago como la fundación de importantes núcleos urbanos como Estella, lograron aumentar considerablemente la circulación monetaria. Pero quizá más importante que el auge en si de tal circulación, debemos reparar en el tipo de moneda que la protagoniza. Junto a los maravedís procedentes en su mayoria de la ceca de Murcia, se utilizan los solidos merguiles o los solidos morlanes, ambos de procedencia gala $y$, desde luego, solidos navarrensi monete ${ }^{55}$. Ello nos hace pensar en un extraordinario desarrollo de las comunicaciones, no sólo en el interior del reino de Navarra, sino particularmente en su conexión con el resto de la Península y, desde luego, con todo ese mundo que hizo del Camino de Santiago la arteria fundamental de enlace con ésta.

Finalmente, cabría reseñar una serie de trabajos que, aunque en principio dedicados a materias completamente ajenas a las vías de comunicación, nos

52 Cuya más reciente labor de síntesis y clasificación de sus series cabe atribuir a J. F. UTRILLA, «Tradición manuscrita, redacciones y edición crítica del Fuero General de Navarra", Principe de Viana. Primer Congreso General de Historia de Navarra, XLIX, 1988, pp. 647-656; «E] Fuero General de Navarra: estudio y edición de las redacciones protosistemáticas (Series A y B)», Pamplona, 1987; Vid. asimismo Martín DuQue, A. J. "Sancho VI de Navarra y el Fuero de Vitoria", Congreso de Estudios Históricos. Vitoria en la Edad Media, Vitoria, 1982; SALINAS QUIJADA, F. «Estudios de Historia de Derecho Foral de Navarra», Pamplona, 1978; LACARRA, J. M. y MARTín DuQUE, A. J. «Fueros de Navarra, 1. Fueros derivados de Jaca, 2. Pamplona», Pamplona, 1975; ForTuN, L. J. "Colección de "fueros menores" de Navarra y otros privilegios locales", Príncipe de Viana, 165, 1982, pp. 296-298; Garcia Gallo, A. "Los fueros de Medinaceli", Anuario de Historia del Derecho Español, 1961, 31, pp. 9-16; Ramos Loscertales, J. M. "Fuero de Jaca», Barcelona, 1927; Id. "Los fueros de Sobrarbe", Cuadernos de Historia de España, VII, 1947, pp. 35-66; Fortun PÉREz DE CIRIzA, L. J. "Los fueros de unificación de pechas de Sancho el Sabio", Vitoria en la Edad Media, Vitoria, 1982, pp. 525-532.

53 LÓPEZ BELTRÁN, M. T. «Economía y Derecho: el molino en los fueros del Valle del Ebro», Hispania, CLIII, Madrid, 1983, pp. 5-22.

54 Zamanillo ARIZABalo, $M^{a}$ A. "Circulación monetaria y sistemas de pago en Navarra en los siglos $x$ a XIII", Príncipe de Viana. Primer Congreso General de Historia de Navarra, XLIX, 1988, pp. 239-245.

55 Lacarra, J. M. Colección Diplomática de Irache, vol. I (958-1222), Nº3, año 1111. 
ofrecen datos enormemente interesantes para nuestro estudio. Cuendo Villegas y Toledano ${ }^{56}$ intentan aportar noticias acerca de la industria textil en Navarra, lo hacen a partir de la documentación que se conserva sobre el viaje de Ponz de Eslava a Zaragoza en $1372{ }^{57}$ buscando el emplazamiento adecuado para instalar una industria textil de alta calidad. De esa forma nos proporcionan las etapas del viaje ${ }^{58}$. No es necesario indicar que tal viaje ha sido estudiado desde el punto de vista económico exclusivamente, razón por la que sería apreciable la aportación geográfica del mismo. Algo parecido ocurre con los estudios de García de Cortázar sobre el equipamiento molinar en la Rioja Alta ${ }^{59}$, de E. Cantera sobre la geografía histórica de la Rioja ${ }^{60}$, de Garcia Guatas sobre el castillo de Avizanda ${ }^{61}$, 0 de Martinez Ochoa acerca de la ordenación territorial ${ }^{62}$, en los que, a través de distintas perspectivas, se nos permite extraer datos de enorme importancia para nuestra reconstrucción.

A la vista de todo lo anterior podemos concluir refiriéndonos al escaso material bibliográfico específico con que contamos para llevar a cabo el estudio propuesto en la etapa señalada. Con las lógicas precauciones, podríamos ofrecer una primera aproximación cartográfica del espacio navarro entre los siglos VIII y XII a partir de las obras comentadas y siempre a la espera de completar la información con los datos pormenorizados que ofrece la documentación. Sin embargo, no es este el objeto del presente trabajo, que únicamente aspira a ofrecer un repertorio bibliográfico general que estimamos como punto de partida para una posterior investigación.

56 Villegas Díaz, L. R. y TOledano Galera, J. «Datos sobre la industria textil en Navarra en el siglo XIV», Príncipe de Viana. Primer Congreso General de Historia de Navarra, XLIX, 1988, pp. $669-676$.

57 A.G.N. Sec. Comptos, Docs. caja $24, n^{\circ} 41$.

58 Zaragoza, Pamplona, Puente la Reina, Estella, Olite, Falces y Tudela.

59 García de Cortazar, J. A. “El equipamiento molinar en la Rioja Alta en los siglos x al XIII", Homenaje a Fray Justo Pérez de Urbel, 1, Silos, pp. 387-405.

${ }^{60}$ Cantera Montenegro, E. "Notas para un estudio de geografía histórica de La Rioja en la Edad Media: núcleos de población en La Rioja Alta a mediados del siglo xIII». En la España Medieval. Estudios en memoria del profesor D. Claudio Sánchez Albornoz V, vol.l, Madrid, 1986, pp. 245-265.

61 Garcia GuAtas, M. "El castillo de Abizanda, en la frontera de la reconquista aragonesa". Homenaje a Don José Maria Lacarra de Miguel en su jubilación del profesorado, I, Zaragoza, 1977, pp. 121-134.

62 Martinez Ochoa, R. M. "La ordenación del territorio en la más vieja Castilla en los siglos ix al x|». Boletín de la linstitución Fernán González, 189/90, 1977/78, pp. 174-339; 81-117. 